\title{
Cerebellar timing and negative patterning
} Horatiu Voicu

Address: Department of Neurobiology and Anatomy, University of Texas Health Science Center, Houston, TX, 77030, USA

Email: Horatiu Voicu - horatiu@voicu.us

from Seventeenth Annual Computational Neuroscience Meeting: CNS*2008

Portland, OR, USA. 19-24 July 2008

Published: II July 2008

BMC Neuroscience 2008, 9(Suppl I):PI doi:I0.1/86/I47I-2202-9-SI-PI

This abstract is available from: http://www.biomedcentral.com/I47I-2202/9/SI/PI

(c) 2008 Voicu; licensee BioMed Central Ltd.

Experimental studies suggest that the rabbit cerebellum is able to show negative patterning (exclusive or), a paradigm in which the rabbit eyelid is conditioned to respond to two individual stimuli, but not to both stimuli presented together. The paradigm, however, works only for interstimulus intervals (ISIs) longer than $800 \mathrm{~ms}$. In this study we use a large scale simulation of the cerebellum to investigate the dynamics between cerebellar timing and the negative patterning paradigm at shorter ISIs. Since the simulation is not able to learn negative patterning with the standard paradigm we design a modified negative patterning training paradigm which is able to produce the standard results. The training mechanism induced by the new paradigm suggests that cerebellar timing and the negative patterning paradigm are mutually exclusive for ISIs shorter than $800 \mathrm{~ms}$. 